• 保护论坛・

\title{
国家管辖范围外海域遗传资源获取和 惠益分享机制构建建议
}

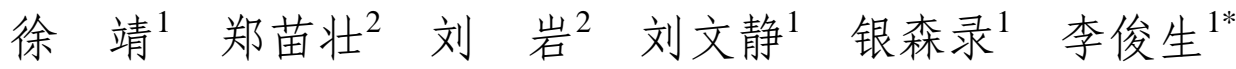 \\ 1 (中国环境科学研究院生物多样性研究中心, 北京 100012) \\ 2 (国家海洋局海洋发展战略研究所, 北京 100860)
}

\begin{abstract}
摘要: 随着人类对海洋资源开发利用强度的增加, 海洋生物多样性的养护面临着更大挑战。《联合国海洋法公约》 及其执行协定已不能较好地应对这些挑战。为此, 联合国大会决定成立工作组, 拟订新的国际协定, 以解决国家管 辖范围外海域生物多样性的养护与可持续利用问题。建立国家管辖范围外海洋遗传资源的获取和惠益分享机制是 该议题的焦点问题之一, 但是目前各国对新的国际执行协定应当包含哪些要素还缺乏共同理解。本文通过分析《生 物多样性公约》、 《名古屋议定书》及《粮食和农业植物遗传资源国际条约》等与遗传资源获取和惠益分享相关 的国际条约在遗传资源的定义、管辖范围、获取条件及惠益分享要求等方面的异同, 提出对海洋遗传资源获取和 惠益分享机制的构建建议: (1)国家管辖范围外的海洋遗传资源不可自由获取; (2)海洋遗传资源的定义应当涵盖海 洋生物代谢生成的海洋天然产物; (3)惠益分享责任适用于在新执行协定生效前获取, 但在其生效后进行开发利用 的遗传资源; (4)获取活动都应得到主管当局批准, 并签订材料转让协议或共同商定条件; (5)应为非商业化的获取 活动制定简化程序, 在人类健康、粮食安全等受到重大威胁而急需获取海洋遗传资源时, 也应适用简化程序, 但后 续商业化活动须分享惠益; (6)针对海洋遗传资源的不同研发环节, 设置惠益分享类型, 并就后续利用进行披露和 监测, 确保惠益得到分享。
\end{abstract}

关键词: 生物多样性; 名古屋议定书; 海洋法; 海洋遗传资源; 生物勘探

\section{Establishment of a mechanism on sharing the benefits arising from the utilization of marine genetic resources in areas beyond national jurisdic- tion}

Jing Xu${ }^{1}$, Miaozhuang Zheng ${ }^{2}$, Yan Liu ${ }^{2}$, Wenjing Liu ${ }^{1}$, Senlu Yin ${ }^{1}$, Junsheng $\mathrm{Li}^{{ }^{*}}$

1 Biodiversity Research Center, Chinese Academy of Environmental Sciences, Beijing 100012

2 Chinese Institute for Marine Affairs, State Oceanic Administration, Beijing 100860

\begin{abstract}
Along with the increase in the utilization of marine biodiversity, conservation is facing challenges. The United Nations Convention on the Law of the Sea and its protocols cannot overcome these challenges. The United Nations General Assembly has mandated a working group with the aim to elaborate a new protocol, in order to address the conservation and sustainable use of biodiversity beyond national jurisdiction. Access to marine genetic resources in areas beyond national jurisdictions and sharing of benefits arising from their utilization is one of the core issues in discussion, but there is also a lack of common understanding among UN member states regarding the elements of this new protocol. In this paper, we analyze the differences and commonalities of definitions, scope, access, and benefit-sharing in the Convention on Biological Diversity, the Nagoya Protocol, and the International Treaty on Plant Genetic Resources for Food and Agriculture. Recommendations for the establishment of access and benefit-sharing mechanisms for marine genetic resources in areas beyond national jurisdictions were raised: (1) marine genetic resources in areas be-
\end{abstract}

收稿日期: 2015-06-08; 接受日期: 2015-12-25

基金项目：环境保护部自然生态保护司生物多样性国际合作及履约项目“《生物多样性公约》国际热点谈判问题追踪研究”(2024004001001); 国家海洋 局海洋发展战略研究所委托课题“国家管辖范围以外区域海洋遗传资源问题研究” (5215002008)

* 通讯作者 Author for correspondence. E-mail: Ljsh@craes.org.cn 
yond national jurisdictions are not free to access; (2) the definition of marine genetic resources should cover marine natural products, which are derived from the metabolism of marine living organisms; (3) benefit-sharing obligation shall apply to the genetic resources are accessed before the entry into force of new protocol but are utilized after that; (4) access should be authorized by a competent authority to ensure an Material Transfer Agreement or mutually agreed terms are established; (5) simplified procedures should apply to access for noncommercial purpose, and to great threats on health and food safety, but benefits should also be shared if there is a follow-up commercialization plan; and (6) benefits should be clarified at different stages of the R\&D chain, as disclosure of information and monitoring of follow-up utilization conducts are necessary to ensure benefits are shared.

Key words: biodiversity; the Nagoya Protocol; the law of the sea; marine genetic resources; bio-prospecting

国家管辖范围外海域占全球海洋面积的 $64 \%$ 。 随着科技的进步，人类对海洋资源的开发利用强度 增加。深海海底矿产和碳烃化合物的大规模开采、 渔业捕捞及气候变化等因素, 均对深海生物多样性 形成威胁(Jobstvogt et al, 2014)。各类海洋资源使用 者同时进入外海, 增加了国家管辖外海域的开发利 用强度(Merrie et al, 2014)。

目前，国家管辖范围外海域的治理机制还不完 善(Freestone et al, 2014)。《联合国海洋法公约》已 通过《关于执行1982年12月10日<联合国海洋法公 约>第十一部分的协定》(http://www.un.org/Depts/ los/convention_agreements/texts/unclos/closindxAgree. $h t m$ )和《执行1982年<联合国海洋法公约>有关养护 和管理跨界鱼类种群和高度洄游鱼类种群的规定 的协定》 (http://www.un.org/Depts/los/convention agreements/texts/fish_stocks_agreement/CONF164_ 37.htm), 分别对矿产开发和渔业捕捞两个问题进行 了规定。但该公约尚未通过任何执行协定对深海遗 传资源的开发进行监管。

2004年，联合国大会决定设立不限成员名额特 设工作组, 研究国家管辖范围外海域生物多样性的 养护和可持续利用问题(UNGA，2004)。2006-2014 年, 联合国大会授权成立的特设工作组先后召开8 次会议, 对《联合国海洋法公约》框架下海洋遗传 资源(marine genetic resources, MGRs)的获取和惠益 分享、公海保护区及环境影响评价等与国家管辖范 围外海域生物多样性的养护和可持续利用相关问 题进行了讨论。2012年, 联合国可持续发展峰会 $(R i o+20)$ 要求联合国大会于2015年前在《联合国海 洋法公约》框架内制定旨在解决国家管辖范围外海 域生物多样性的养护和可持续利用问题的新协定 (UNGA, 2012)。
MGRs的保护和可持续利用是联合国大会新执 行协定中的核心问题(Leary et al, 2009; Druel \& Gjerde, 2014)。当前《联合国海洋法公约》讨论该 问题的主要障碍是缺乏对遗传资源获取和惠益分 享模式的了解和研究。本文通过分析与MGRs相关 的《生物多样性公约》(the Convention on Biological Diversity, CBD; 以下简称《公约》)、《遗传资源获 取与惠益分享的名古屋议定书》(the Nagoya Protocol on Access to Genetic Resources and the Fair and Equitable Sharing of Benefits Arising from Their Utilization to the Convention on Biological Diversity, $\mathrm{NP}$; 以下简称《名古屋议定书》)及《粮食和农业植 物遗传资源国际条约》(the International Treaty on Plant Genetic Resources for Food and Agriculture, ITPGRFA; 以下简称《粮农条约》)等与遗传资源获 取和惠益分享相关的国际条约在遗传资源的定义、 管辖范围、获取条件及惠益分享要求等方面的异同 和经验, 同时结合MGRs获取和惠益分享的特点, 提出在国家管辖范围外海域MGRs获取和惠益分享 机制的构建建议。

\section{1 相关国际法律文书基本要素的异同}

\section{1 遗传资源的定义}

《公约》与《粮农条约》的定义中所称遗传功 能单位仅指核酸及经遗传表达产生的蛋白质或酶, 而《名古屋议定书》对遗传资源的定义有较大范围 的扩展(Box 1), 不仅包含核酸及经遗传表达产生的 蛋白质或酶, 也涵盖了各种自然产生的生物化学化 合物(徐靖等, 2012, 2013b)。

\section{2 文书的管辖范围}

管辖范围包含时间、地理及对象范围。《公约》、 《名古屋议定书》及《粮农条约》均未对时间范围 
作出规定, 这是《名古屋议定书》的明显不足(薛达 元, 2011)。目前, 非洲诸国正推动《名古屋议定书》 第10条全球多边惠益分享机制的讨论, 以解决《公 约》或《名古屋议定书》生效前获取遗传资源的惠 益分享问题(徐靖等, 2013a)。此外, 3个文书也未明 确提出各自的地理范围, 仅对管辖对象进行了各自 表述。其中, 《公约》与《名古屋议定书》对管辖 对象的表述较为模糊, 《粮农条约》的管辖对象相对 清晰(Box 1)。

\section{3 遗传资源的获取}

在权属方面，《公约》、《名古屋议定书》及《粮 农条约》均确认各国对其自然资源拥有主权, 但《粮 农条约》更加强调缔约方应便利遗传资源的获取。 在获取审批方面, 《公约》及《名古屋议定书》侧 重于对商业化获取行为进行管理, 《粮农条约》侧 重保障以粮食安全为目的的非商业化获取活动。关 于获取条件, 《公约》及《名古屋议定书》规定国 家有权审查和否决未依法拟订的转让协议, 《粮农 条约》则要求提供者和获取者使用由国际公认的标 准材料转让协议。在获取成本上, 《公约》未做明 确规定, 《名古屋议定书》明确了获取和提供者双 方可以议定获取成本, 而《粮农条约》出于粮食安 全考虑，强调最低成本的资源获取(Box 1)。

\section{4 遗传资源利用及其惠益分享}

关于惠益分享类型, 与《公约》和《名古屋议 定书》相比, 《粮农条约》中的惠益分享类型多为 非货币性质, 但同时规定, 若获取者利用从多边体 系获取的材料培育出了新的商业化产品, 则须将其 商业化后总销售额的 $1.1 \%$ 捐献给《粮农条约》。关 于惠益分享的受益群体, 《名古屋议定书》采用了 “双边模式” (徐靖等, 2013a), 惠益可由遗传资源提 供者代表缔约方享有。《粮农条约》的惠益则由国 家享有。关于协议的遵守, 《名古屋议定书》要求 缔约方监测国内的遗传资源开发利用活动, 《粮农 条约》则明确提出无须对提供的资源进行跟踪。在 争端解决方面, 《公约》及《粮农条约》倾向于在 多边体系内解决问题, 《名古屋议定书》则倾向于 按照获取协议中约定的争端解决方式进行解决 (Box 1)。

\section{2 对新执行协定谈判的建议}

国家管辖范围外海域的MGRs绝不意味着可以
自由获取。在国家管辖范围外海域, 国家对海洋生 物资源并不享有主权权利, 各项监管制度也未建 立，因此各国普遍缺乏保护意识，倾向于“先到先 得”。如果资源使用者只有开发利用的权利而没有 保护的义务，这必然会导致“公地悲剧”(Hardin, 1968)。因此，建立公平公正地分享由MGRs开发利 用产生惠益的国际执行协定, 是非常迫切和必要 的。下面就新执行协定的谈判提出 4 个方面的建议。

\section{1 明确海洋遗传资源的定义}

海洋天然产物(marine natural product)是海洋生 物资源利用的重要对象。20世纪50年代首次分离出 海洋生物活性化合物海绵尿苷及海绵胸苷, 后被证 明具有抗癌和抗病毒功效(Leary et al, 2009)。其后 30年间, 宏基因组文库及全基因靶标测序等技术的 出现, 使海洋生物次级代谢产物研究得到了发展。 Leary等(2009)研究了1999-2004年间报道海洋天然 产物具有抗肿瘤和细胞毒性特征文章的期刊数量 及种类, 发现刊文数量并发现数量呈递增趋势, 且 主要为聚酮化合物、萜类化合物、含氮化合物及多 糖。自20世纪90年代以来, 全球已申报MGRs专利 4,900 余种, 发现相关自然产物 17,000余种, 这些发 现主要集中于多孔动物门、刺胞动物门及红藻门物 种(Arrieta et al, 2010; Leal et al, 2012)。2004年以来, 美国、欧盟等先后批准了一批源于海洋天然产物的 药物进入市场, 而其他国家也正在推动海洋天然产 物药物的产、学、研合作(Molinski et al, 2009)。

因此, 基于MGRs开发的生物药的销售利润是 国家管辖范围外MGRs的潜在惠益之一。新执行协 定可以借鉴《名古屋议定书》经验, 就MGRs定义是 否涵盖海洋生物代谢生成的海洋天然产物进行深 入讨论。新执行协定还可以参考《名古屋议定书》 的工作基础, 分别就 “MGRs”及 “利用 MGRs”(the utilization of MGRs)进行定义。

\section{2 确定执行协定的管辖范围}

国际法律文书的管辖范围越清晰, 在实践中与 其他国际法律文书的重叠和冲突的可能性越小。 “法不溯及既往”原则虽应在联合国大会有关MGRs 讨论进程中得到了反映, 但惠益分享的义务究竟是 以获取行为发生时间还是以开发利用活动发生时 间计算, 是应当解决的问题。目前, 一些国家已对 来自公海的MGRs进行了收集和保藏，这些资源尚 未得到很好的开发利用, 但不排除它们在新执行协 


\begin{tabular}{|c|c|c|c|}
\hline \multicolumn{4}{|c|}{ 辖范围、获取条件及惠益分享要求的比较 } \\
\hline & 《生物多样性公约》(CBD) & 《名古屋议定书》(NP) & 《粮农条约》(ITPGRFA) \\
\hline \multicolumn{4}{|l|}{ 定义 } \\
\hline 遗传资源 & 具有实际或潜在价值的遗传材料 & 与CBD相同 & $\begin{array}{l}\text { 对粮食和农业具有实际或潜在价值 } \\
\text { 的任何植物遗传材料 }\end{array}$ \\
\hline 遗传材料 & $\begin{array}{l}\text { 来自植物、动物、微生物或其他来 } \\
\text { 源的任何含有遗传功能单位的材 } \\
\text { 料 }\end{array}$ & 与CBD相同 & $\begin{array}{l}\text { 任何植物源材料, 包括含有遗传功 } \\
\text { 能单位的有性和无性繁殖材料 }\end{array}$ \\
\hline 衍生物 & - & $\begin{array}{l}\text { 由生物或遗传资源的遗传表达或新陈 } \\
\text { 代谢产生的、自然生成的生物化学化 } \\
\text { 合物, 即使其不具备遗传功能单元 }\end{array}$ & - \\
\hline 利用遗传资源 & - & $\begin{array}{l}\text { 对遗传资源的遗传和(或)生物化学组 } \\
\text { 成进行研究和开发, 包括通过应用 } \\
\mathrm{CBD} \text { 第2条定义的生物技术 }\end{array}$ & - \\
\hline 生物技术 & $\begin{array}{l}\text { 使用生物系统、生物体或其衍生物 } \\
\text { 的任何技术应用, 以制作或改进 } \\
\text { 特定用途的产品或工艺过程 }\end{array}$ & 与CBD相同 & - \\
\hline \multicolumn{4}{|l|}{ 管辖范围 } \\
\hline 时间范围 & - & - & - \\
\hline 地理范围 & $\begin{array}{l}\text { (1)国家管辖范围之内的区域(针对 } \\
\text { 生物多样性组成部分) } \\
\text { (2)国家管辖范围以外的区域(针对 } \\
\text { 过程和活动) }\end{array}$ & 与CBD相同 & - \\
\hline 对象范围 & $\begin{array}{l}\text { (1)生物多样性组成部分 } \\
\text { (2)在缔约方管辖或控制下开展的 } \\
\text { 过程和活动 }\end{array}$ & $\begin{array}{l}\text { (1) CBD第15条范围内的遗传资源和 } \\
\text { 利用此种遗传资源所产生的惠益 } \\
\text { (2)CBD范围内的与遗传资源相关的传 } \\
\text { 统知识和利用此种知识所产生的惠益 }\end{array}$ & $\begin{array}{l}\text { ITPGRFA 附录I中包含的粮食和农 } \\
\text { 业植物遗传资源 }\end{array}$ \\
\hline $\begin{array}{l}\text { CBD 第 } 15 \text { 条 } \\
\text { “遗传资源的取 } \\
\text { 得”第3款 }\end{array}$ & $\begin{array}{l}\text { 缔约方所提供的遗传资源, 仅限 } \\
\text { 于这种资源原产国的缔约方或按 } \\
\text { 照本公约取得该资源的缔约方所 } \\
\text { 提供的遗传资源 }\end{array}$ & 与CBD相同 & - \\
\hline \multicolumn{4}{|l|}{ 获取条件 } \\
\hline 对获取的批准 & $\begin{array}{l}\text { 遗传资源的取得须经过提供这种 } \\
\text { 资源的缔约方事先知情同意 }\end{array}$ & $\begin{array}{l}\text { 按照国家立法并经过提供这种资源的 } \\
\text { 缔约方事先知情同意 }\end{array}$ & $\begin{array}{l}\text { 各缔约方同意采取必要的法律措施 } \\
\text { 或其他适当措施, 通过多边系统向 } \\
\text { 其他缔约方及缔约方管辖范围内的 } \\
\text { 自然人和法人提供获取的机会 }\end{array}$ \\
\hline 获取条件 & 根据共同商定条件 & 商业化和非商业化利用 & $\begin{array}{l}\text { 只为粮食和农业研究、育种和培训 } \\
\text { 而利用及保存提供获取机会; 对多 } \\
\text { 用途(食用、非食用)作物的获取, 首 } \\
\text { 先考虑对粮食安全的重要性 } \\
\text { 根据标准的材料转让协议 }\end{array}$ \\
\hline 获取成本 & - & $\begin{array}{l}\text { 可包括转让费和预付费, 按共同商定 } \\
\text { 条件执行 }\end{array}$ & $\begin{array}{l}\text { (1)原则上无偿提供 } \\
\text { (2)如收取费用, 则不得超过所涉及 } \\
\text { 的最低成本 }\end{array}$ \\
\hline \multicolumn{4}{|l|}{ 惠益分享要求 } \\
\hline 惠益类型 & - & $\begin{array}{l}\text { 货币性和非货币性惠益，可包括知识 } \\
\text { 产权的分享 }\end{array}$ & $\begin{array}{l}\text { 便利获取资源及相关信息、技术、 } \\
\text { 能力建设。知识产权的申请须强制 } \\
\text { 惠益分享 }\end{array}$ \\
\hline 主要受益群体 & - & 资源提供者代表缔约方受益 & $\begin{array}{l}\text { 缔约方通过实施滚动式《粮食和农 } \\
\text { 业植物遗传资源保存及可持续利用 } \\
\text { 全球行动计划》受益 }\end{array}$ \\
\hline $\begin{array}{l}\text { 利 用 活动 是否 } \\
\text { 需要监测 }\end{array}$ & - & 由(使用者所在)缔约方监测 & 无须跟踪单份收集品 ～～～～～～～～～～ \\
\hline 争端解决机制 & $\begin{array}{l}\text { 谈判、第三方斡旋或调解, 仲裁和 } \\
\text { 上诉国际法庭等 }\end{array}$ & 共同商定条件含有争端解决条款 & 与CBD相同 \\
\hline
\end{tabular}


定生效后得到开发利用。从时间节点上看, 此类 MGRs的获取行为虽发生在新协定生效前, 但其开 发利用行为发生在新协定生效之后, 若以开发利用 活动发生时间确定惠益分享责任, 这类研发活动无 疑需要分享惠益。

因此，联合国大会关于MGRs问题的讨论中可 以借鉴《名古屋议定书》第10条关于来源不明、无 法获得事先知情同意的遗传资源惠益分享问题的 思路和经验，应明确使用者承担惠益分享责任的时 间起点是对MGRs的开发利用而非获取的起始时间, 将MGRs分为已知从国家管辖范围以外海域获取的 MGRs和未知来源的MGRs, 以及在新执行协定或 《联合国海洋法公约》生效前获取并保存在菌种保 藏库中的MGRs。

\section{3 制定海洋遗传资源的获取条件}

确定MGRs的所有权人是为国家管辖范围以外 的MGRs建立获取和惠益分享机制的基础和关键。 缔约方对国家管辖范围外海域的资源并无主权或 管辖权(郑苗壮等, 2015), 若确有必要对这一部分资 源建立获取和惠益分享机制, 则应借鉴《公约》、《名 古屋议定书》和《粮农条约》的经验。首先, 应建 立事先知情同意制度, 所有MGRs的获取活动都应 事先向有关主管当局提出申请或备案; 获取者还应 与提供者签订协议, 承诺公平公正地分享惠益。其 次, 为了不阻碍科学研究活动, 可对以科研为目的 获取MGRs制定简化条件。但在签订材料转让协议 或共同商定条件时应当明确, 以科研为目的获取 MGRs的研究成果不得申请专利, 如果申请专利, 则须分享由此产生的惠益。最后，在人类健康、粮 食安全等受到重大威胁而急需获取MGRs时, 也应 适用简化程序, 同时如果之后转化为商业化开发, 也必须分享惠益。

\section{4 设置海洋遗传资源的惠益分享要求}

理解遗传资源研发的可行性、成本、模式以及 有关新产品商业化的途径是制定国家管辖范围外 MGRs获取和惠益分享具体措施的关键(Broggiato et al, 2014)。应当结合MGRs在获取-保藏-科研-开 发-商业化等不同环节的特点, 合理设置惠益分享 内容, 确保其可操作性和灵活性。必要时可仿照《名 古屋议定书》给出的指示性清单, 指导惠益分享实 践。但是, 以食用为目的的捕获物及其传统初级加
工产品仍然属于单纯的商业贸易活动，并不涉及对 MGRs潜在价值的开发，不应在新执行协定框架内 分享惠益。

专利申请是可能出现货币性惠益的标志。《联 合国海洋法公约》缔约方大会应与世界知识产权组 织(World Intellectual Property Organization)加强合 作, 要求其加强关于专利申请的信息交换, 履行披 露义务及公布惠益分享信息。

此外，国家对管辖范围外海域的MGRs不能行 使主权权利，因此国家及遗传资源的提供者并不能 直接享受惠益。目前《名古屋议定书》正就全球多 边惠益分享机制进行讨论，进入该机制的资金将主 要用于全球生物多样性保护和可持续利用(徐靖等, 2013a)。联合国大会应当借鉴《粮农条约》和《名 古屋议定书》第10条的经验，建立一种“多边模式”, 确保产生的惠益能够惠及国家管辖范围外海域生 物多样性保护和可持续利用。

最后，对国家管辖范围外MGRs的开发利用活 动进行监测是确保惠益分享的重要手段。《名古屋 议定书》原则上要求获取者所在缔约方及时报告遗 传资源的开发利用情况，并通过信息交换机制予以 公开。虽然《名古屋议定书》的最后文本忽略了披 露义务, 弱化了设置检查点的要求(薛达元, 2011), 但仍有利于发展中国家维护自身权益。虽然《名古 屋议定书》刚刚生效，有关机制的运作效果还不清 楚，但《联合国海洋法公约》缔约方大会仍可借鉴 其经验。

\section{参考文献}

Arrieta JM, Arnaud-Haond S, Duarte CM (2010) What lies underneath: conserving the ocean's genetic resources. Proceedings of the National Academy of Sciences, USA, 107, 18318-18324.

Broggiato A, Arnaud-Haond S, Chiarolla C, Greiber T (2014) Fair and equitable sharing of benefits from the utilization of marine genetic resources in areas beyond national jurisdiction: bridging the gaps between science and policy. Marine Policy, 49, 176-185.

Druel E, Gjerde KM (2014) Sustaining marine life beyond boundaries: options for an implementing agreement for marine biodiversity beyond national jurisdiction under the United Nations Convention on the Law of the Sea. Marine Policy, 49, 90-97.

Freestone D, Johnson D, Ardron J, Morrison KK, Unger S (2014) Can existing institutions protect biodiversity in areas beyond national jurisdiction? Experiences from two 
on-going processes. Marine Policy, 49, 167-175.

Hardin G (1968) The tragedy of the commons. Science, 162, 1243-1248.

Jobstvogt N, Hanley N, Hynes S, Kenter J, Witte U (2014) Twenty thousand sterling under the sea: estimating the value of protecting deep-sea biodiversity. Ecological Economics, 97, 10-19.

Leal MC, Puga J, Serodio J, Gomes NCM, Calado R (2012) Trends in the discovery of new marine natural products from invertebrates over the last two decades-Where and what are we bioprospecting? PLoS ONE 7, e30580.

Leary D, Vierros M, Hamon G, Arico S, Monagle C (2009) Marine genetic resources: a review of scientific and commercial interest. Marine Policy, 33, 183-194.

Merrie A, Dunn DC, Metian M, Boustany AM, Takei Y, Elferink AO, Ota Y, Christensen V, Halpin PN, Osterblom H (2014) An ocean of surprises-trends in human use, unexpected dynamics and governance challenges in areas beyond national jurisdiction. Global Environmental Change, 27, 19-31.

Molinski T, Dalisay D, Lievens S, Saludes J (2009) Drug development from marine natural products. Drug Discovery, 8, 69-85.

United Nations General Assembly (UNGA) (2004) Resolution on Oceans and the Law of the Sea. UN Doc. A/RES/59/24 of 17 November 2004, Para. 73.

United Nations General Assembly (UNGA) (2012) Resolution Endorsing the Rio+20 Outcome Document "The Future We Want”. UN Doc. A/RES/66/288 of 11 September 2012, Para. 162.

Wang SM, Zhang ZW (2011) The implementation progress on the International Treaty on Plant Genetic Resources for Food and Agriculture. Journal of Plant Genetic Resources, 12, 493-496. (in Chinese with English abstract) [王述民, 张宗文 (2011) 《粮食和农业植物遗传资源国际条约》实 施进展. 植物遗传资源学报, 12, 493-496.]
Xu J, Cai L, Wang AH, Xue DY, Yang QW, Li JS, Yin SL (2013a) The global multilateral benefit-sharing mechanism: a new focus in genetic resources access and benefit-sharing negotiations. Biodiversity Science, 21, 127-131. (in Chinese with English abstract) [徐靖, 蔡蕾, 王爱华, 薛达元, 杨庆 文, 李俊生, 银森录 (2013a) 全球多边惠益分享机制: 遗 传资源获取和惠益分享谈判的新焦点. 生物多样性, 21, 127-131.]

Xu J, Li JS, Xue DY, Yin SL (2012) Core contents interpretation of Nagoya Protocol on Genetic Resources Access and Benefit-Sharing and the prediction of its entry into force. Journal of Plant Genetic Resources, 13, 720-725. (in Chinese with English abstract) [徐靖, 李俊生, 薛达元, 银森 录 (2012) 《遗传资源获取与惠益分享的名古屋议定书》 核心内容解读及其生效预测. 植物遗传资源学报, 13 , 720-725.]

Xu J, Yin SL, Li JS (2013b) The comparison of the International Treaty on Plant Genetic Resources for Food and Agriculture and the Nagoya Protocol. Journal of Plant Genetic Resources, 14, 1096-1101. (in Chinese with English abstract) [徐靖, 银森录, 李俊生 (2013b) 《粮食和农业植物 遗传资源国际条约》与《名古屋议定书》比较研究. 植物 遗传资源学报, 14, 1096-1101.]

Xue DY (2011) Analysis for the main elements and potential impacts of Nagoya Protocol. Biodiversity Science, 19, 113-119. (in Chinese with English abstract) [薛达元 (2011) 《名古屋议定书》的主要内容及其潜在影响. 生物多样 性, 19, 113-119.]

Zheng MZ, Liu Y, Xu J (2015) Study on the Convention on Biological Diversity and biodiversity beyond national jurisdiction issue. Journal of China Marine University: Social Sciences, (02), 40-45. (in Chinese) [郑苗壮, 刘岩, 徐靖 (2015) 《生物多样性公约》与国家管辖范围以外海洋生 物多样性问题研究. 中国海洋大学学报(社会科学版), (02), 40-45.] 